\title{
La psicoterapia de resolución de problemas fue tan eficaz como los antidepresivos en la depresión en atención primaria
}

Randomized controlled trial of problem solving treatment, antidepressant medication, and combined treatment for major depression in primary care. Mynors Wallis L M, Gath D H, Day A, Baker, F. BMJ 2000;320:26-30.

\section{Objetivo}

Determinar si la terapia para resolver problemas (TRP) combinada con antidepresivos (AD) es más eficaz que ambos tratamientos por separado en el manejo de la depresión en Atención Primaria (AP)

\section{Diseño}

Ensayo clínico aleatorizado controlado, con 4 grupos de tratamiento

\section{Lugar}

Centros de Atención Primaria en Oxfordshire, Reino Unido.

\section{Pacientes}

Pacientes entre 16-65 años derivados por médicos generalistas (MG) con diagnóstico de depresión mayor, definida con una puntuación de 13 o más en 17 ítems de la escala de Hamilton y una duración mínima de la enfermedad de 4 semanas. Se excluyeron pacientes que tuvieran otra enfermedad psiquiátrica previa a la depresión, que estuviesen recibiendo antidepresivos, tratamiento psicológico, o ambos; abuso de drogas o alcohol, daño cerebral, dificultades en el aprendizaje y cualquier enfermedad física que requiriera tratamiento antidepresivo.

\section{Intervención}

Los pacientes que cumplían con los criterios de inclusión fueron aleatorizados (en forma estratificada*) a recibir uno de los cuatro tratamientos: TRP a cargo de médicos de Atención Primaria (MAP) o enfermeras, medicación AD, o combinación de terapia y AD. Las terapias fueron aplicadas por MAP y enfermeras entrenados previamente. Se realizaron 6 sesiones de tratamiento (semana 1,2,3,5,7 y 11). La entrevista inicial fue de una hora, las 5 siguientes de 30 minutos. Como tratamiento antidepresivo los pacientes recibieron fluvoxamina con dosis iniciales de $100 \mathrm{mg}$ y eventualmente modificada a paroxetina (20 mg inicialmente). La dosis fue modificada según el estado clínico y cumplimiento del paciente.

\section{Medición de resultados principales}

Los resultados se midieron a las 6,12 y 52 semanas. Se utilizó la escala de Hamilton y el Inventario de Beck para depresión, como medidas de severidad; y el Protocolo de Entrevista Clínica y Escala social como medida de síntomas psicológicos y funcionamiento social. Se definió paciente recuperado si el puntaje del Hamilton era 7 o menos, parcialmente recuperado con 8 a 12 y no recuperados con puntajes de 13 o más.

\section{Resultados Principales}

Fueron derivados 241 pacientes, de los cuales 151 entraron al estudio y 116 lo completaron (77\%). La media en el número de sesiones del grupo TRP fue 4.6 (rango 1 a 7 ) y 5.2 en el grupo que combinaba TRP y $A D$

TABLA: Número de pacientes recuperados (\%) al final del tratamiento (semana 12) y a las 52 semanas de seguimiento.

\begin{tabular}{l|c|c|c|c|c|c|c|c|}
\hline Recuperación N(\%) & \multicolumn{2}{|c|}{ TRP (MAP) } & \multicolumn{2}{|c|}{ TRP (enfermera) } & \multicolumn{2}{|c|}{ AD } & \multicolumn{2}{|c|}{ TRP y AD } \\
\hline $\begin{array}{l}\text { Recuperación } \\
\text { total }\end{array}$ & $20(51)$ & $24(62)$ & $22(54)$ & $23(56)$ & $24(67)$ & $20(56)$ & $21(60)$ & $23(66)$ \\
\hline $\begin{array}{l}\text { Parcialmente } \\
\text { recuperado }\end{array}$ & $5(13)$ & $6(15)$ & $6(15)$ & $8(20)$ & $4(11)$ & $9(25)$ & $5(14)$ & $5(14)$ \\
\hline \begin{tabular}{l} 
No recuperado \\
\hline
\end{tabular} & $14(32)$ & $9(23)$ & $13(32)$ & $10(24)$ & $8(22)$ & $7(19)$ & $9(26)$ & $7(21)$ \\
\hline
\end{tabular}

TRP terapia para resolver problemas, AD antidepresivos, MAP médicos de atención primaria

Los 4 grupos mostraron mejoría durante el tratamiento y no hubo diferencias significativas entre los mismos. La combinación de terapia con TRP y AD no fue más eficaz que cada una por separada. No hubo diferencia entre la TRP administrada por médicos y enfermeras.

\section{Conclusiones}

La TRP, un tratamiento estructurado de tipo cognitivo, fue efectiva y con resultados similares al tratamiento con $A D$. El tratamiento puede ser efectuado tanto por médicos como por enfermeras de atención primaria entrenados en ese método terapéutico. La combinación de $\mathrm{AD}$ con TRP no aumentó la eficacia.

Fuente de financiamiento: Instituto Nacional del Envejecimiento dependiente de los Institutos Nacionales de la Salud.

\section{Comentario}

Este trabajo demuestra que el tratamiento psicoterapéutico con TRP, en depresión leve y moderada sin comorbilidad, tuvo un resultado similar al tratamiento farmacológico. En relación a la aplicabilidad es importante mencionar que estos resultados no son necesariamente extensivos a otros modelos terapéuticos. Hay evidencia a favor que distintos tratamientos, empíricamente válidos, tienen eficacia similares y que las diferencias en los resultados dependerían más del profesional que del modelo. Debemos señalar que el estudio no evalúa el tratamiento en depresión grave, que de todos modos quedaría excluida del manejo exclusivo de los médicos de atención primaria.

A pesar de que en el presente estudio se realizó un seguimiento a un año, no se tienen datos de recurrencias a largo plazo. En este sentido existe evidencia controvertida al respecto, ya que algunos trabajos consideran que la terapia cognitiva sola o combinada con $\mathrm{AD}$ arroja resultados más duraderos y menor número de recaídas y recidivas que la terapia antidepresiva sola. En relación al resultado principal, se definió como resultado positivo si existía remisión y no solo mejoría o respuesta al tratamien- to. Esto se debe a que la sola mejoría, sin remisión, tiene peor pronóstico a largo plazo.

Los requerimientos del diseño exigieron que los pacientes fueran derivados a profesionales que no son los que tratan habitualmente a los pacientes. De hecho estos pacientes fueron derivados a un "especialista" (en este caso, un médico de atención primaria entrenado en TRP). El tipo de relación de un paciente con el médico que lo atiende con continuidad es muy diferente. Además ese médico formó parte del sistema terapéutico donde el paciente adquirió su depresión; esto es muy importante al momento de generalizar los resultados.

Los profesionales de atención primaria, tanto médicos como enfermeros, pudieron entrenarse adecuadamente en este modelo de psicoterapia; lo que no sabemos es a qué costo y cuáles fueron las exigencias para la inclusión en el programa (interés, competencias, preferencias y habilidades). Deberíamos pensar cuál sería la ventaja en países como la Argentina, donde el recurso humano en psicoterapia es abundante.

Dr. Jorge Bernstein [ Dto. Medicina Familiar, Fac. Medicina, UBA. Serv. Psicopatología, Swiss Medical Group ]

Dra. Mónica V. Santagata [ Coordinadora del Programa de Medicina Transcultural. Hospital Italiano. Psicopatología, OMINT ] 